\title{
THE ROLE OF OCCLUSAL FACTORS IN TEMPORO MANDIBULAR DISEASES - A SYSTEMATIC REVIEW
}

\author{
Sruthi YSS1, Rao BL2, Chakradhar V3, Parvathi PSHL4, Pallavi C $^{5}$
}

\begin{abstract}
1. Senior lecturer, Department of prosthodontics, Lenora institute of dental sciences
2. Professor\& Head, Department of prosthodontics, Lenora institute of dental sciences

3. Postgraduate student, Department of prosthodontics, Lenora institute of dental sciences

4. Postgraduate student, Department of prosthodontics, Lenora institute of dental sciences

5. Senior lecturer, Department of prosthodontics, Sree Sai dental college \& research institute.
\end{abstract}

\begin{abstract}
PURPOSE: The aim of this systematic review is to investigate the role of TMDs and offer a suggestion as to how best to manage them.METHODOLOGY: Databases including Google Scholar, Embase, Scopus, and Medline were accessed between October 1975 to May 2018. Only English language articles were selected. No additional sources were accessed, including gray literature. The topic addressed was the following: interaction between occlusion and TMD' 41 articles were sourced relevant to the research question under discussion. None of the articles were duplicates, while four were discarded after reading the abstract, and 15 articles were excluded after reading the articles in full text for studying other factors along with occlusion in their study. A total, therefore, 22 articles were finally selected and included based on their relevance in addressing the proposed research topic. All of them were full text accessed and qualitatively analyzed results.RESULTS: The articles selected demonstrated that there were three streams of studies. While two of the streams were diametrically opposite to each other regarding their conclusions, the third stream pointed out that the methodological flaws in the studies implied that further studies might be needed to arrive at a definite conclusion.CONCLUSION: This systematic review arrived at the following conclusions:1. A majority of studies do not find occlusion as an aetiologic role in TMD; 2.there are a few studies which, however, find an association, and it is the duty of the clinician to differentiate and diagnose the different entities of temporomandibular disorders and adjust the treatment modality accordingly.
\end{abstract}

KEYWORDS: Occlusion, Temporo-mandibular disorders (TMD), Cranio-mandibular disorders. 
INTRODUCTION:

Temporomandibular disorders (TMDs) have been and continues to be a subject of controversy' even today; this can be attributed to two reasons. One lack of clarity with regards to the functioning of the Temporomandibular joint (TMJ) and the other to the inability to pinpoint a definite etiology for the condition the latter is, however, not due to shortage of aetiologies for the condition but rather due to the plethora of such aetiologies but among the many aetiologies, one has been a significant source of dissonance.1,2 It is the role of occlusion in TMDs. This one cause has been investigated by many over a long period of time, but the conclusions have not been conclusive divergent results have emerged, which, rather than offering clarity, have often served to cloud the picture. Reviews, including systematic reviews, have also not helped because they usually end up either confirming or denying the role of occlusion in TMDs.

This review is, therefore, an attempt to present both the divergent viewpoints as well as a third one which is that further studies are required since many of the studies are flawed methodologically. This systematic review will, therefore, present all three aspects, thus enabling the reader to get a balanced view and help promote further investigation into the topic. A hand search was initiated by the third author, and the keywords used in the hand search were occlusion, Temporo-mandibular disorders (TMD), Cranio-mandibular disorders.

\section{DATA SOURCES:}

Databases including Google Scholar, Embase, Scopus, and Medline were accessed between October 1975 to May 2018, using the keywords Occlusion, Temporo-mandibular disorders (TMD), and Cranio-mandibular disorders. Only English language articles were selected. No additional sources were accessed, including gray literature. The question addressed was the following: what is the association between occlusion and TMD' 41 articles were sourced relevant to the research question under discussion. None of the articles were duplicates, while four were discarded after reading the abstract, and 15 articles were excluded after reading the articles in full text for studying other factors along with occlusion in their study. A total, therefore, 22 articles were finally selected and included based on their relevance in addressing the proposed research question. All of them were full text accessed and qualitatively analyzed results. The chosen articles demonstrated that there were three streams of studies.

While two of the streams were diametrically opposite to each other regarding their conclusions, the third stream pointed out that the methodological flaws in the studies implied that further studies might be needed to arrive at a definite conclusion. 


\section{FLOW CHART 1: PRISMA flowchart for selection of the} article

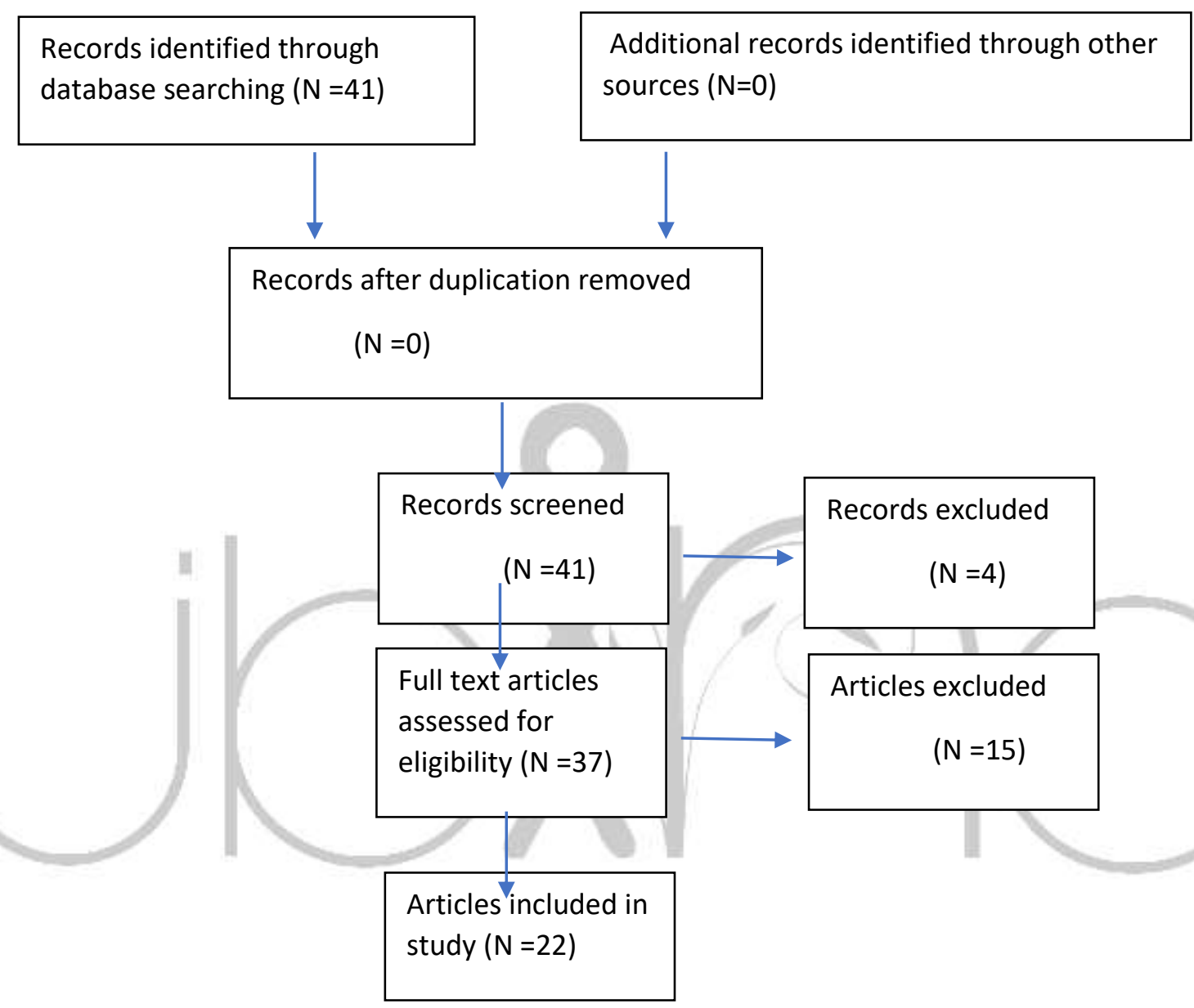

Table 1 excluded articles and the reasons for exclusion :

\begin{tabular}{|l|l|l|}
\hline S.NO & $\begin{array}{l}\text { FIRST AUTHOR \& } \\
\text { YEAR }\end{array}$ & REASONS FOR EXCLUDING \\
\hline 1 & Iger .egermartx ${ }^{3} 1992$ & $\begin{array}{l}\text { Not relevant to the research question as it examined the } \\
\text { relationship between orthodontic patients and TMD }\end{array}$ \\
\hline 2 & Peter Fuchs ${ }^{4} 1975$ & $\begin{array}{l}\text { The study investigated the nocturnal muscle activity of the } \\
\text { stomatognathic system. Hence unrelated to the research } \\
\text { question }\end{array}$ \\
\hline
\end{tabular}




\begin{tabular}{|c|c|c|}
\hline 3 & Lotika Wadhwa 1993 & $\begin{array}{l}\text { The study examined the role of orthodontics in TMD and } \\
\text { hence unrelated to the research question }\end{array}$ \\
\hline \begin{tabular}{|l|l} 
\\
\end{tabular} & M.J Trenouth ${ }^{6} 1979$ & $\begin{array}{l}\text { The study was on the relationship between TMD and } \\
\text { bruxism hence not relevant to the research question }\end{array}$ \\
\hline 5 & PullingerAJ ${ }^{7} 1993$ & $\begin{array}{l}\text { The study was to find the role of attrition, TMD. occlusal } \\
\text { factors were not taken into considerations }\end{array}$ \\
\hline 6 & H.G. String & $\begin{array}{l}\text { Excluded because the study dealt with variations in } \\
\text { skeletal and dental patterns in joints and co-relation with } \\
\text { TMD not specific to the research question }\end{array}$ \\
\hline 7 & Risse $\mathrm{C}^{9} 1984$ & $\begin{array}{l}\text { The study investigated occlusal interferences with certain } \\
\text { muscle groups activity }\end{array}$ \\
\hline 8 & Pullinger $\mathrm{A}^{10} 1991$ & $\begin{array}{l}\text { Studied overjet }+ \text { bite relations with osteoarthritis and } \\
\text { hence not relevant to the research question }\end{array}$ \\
\hline 9 & $\operatorname{Rugh}^{11} 1984$ & $\begin{array}{l}\text { The study examined the role of experimental occlusal } \\
\text { discrepancies on nocturnal bruxism and hence not } \\
\text { relevant to the research question }\end{array}$ \\
\hline 10 & WI & $\begin{array}{l}\text { The study was restricted to adolescents and hence was age } \\
\text { limited in scope }\end{array}$ \\
\hline 11 & Tsolka $\mathrm{P}^{13} 1995$ & $\begin{array}{l}\text { The study was to examine the occlusal variables and } \\
\text { bruxism on TMD. Hence not specific to the research } \\
\text { question }\end{array}$ \\
\hline 12 & Suvinen ${ }^{14} 2007$ & EMG studies, occlusal factors. \\
\hline 13 & Luther $\mathrm{F}^{15} 2007$ & This article addressed the role of orthodontics in TMD \\
\hline 14 & TamneK ${ }^{16} 1993$ & $\begin{array}{l}\text { The study investigated the association between } \\
\text { malocclusion and TMD in orthodontic patients }\end{array}$ \\
\hline 15 & MarbachJJ ${ }^{17} 1990$ & $\begin{array}{l}\text { The study assessed the association between tooth grinding } \\
\text { and TMD. So not relevant }\end{array}$ \\
\hline
\end{tabular}

SYSTEMATIC ASSESSMENT OF PAPERS:

The selected papers were analyzed by adhering to the PICOT format. i.e P-population,I-intervention,C - comparison O-
Population - refers to the target population who were part of the study. viz. the users in the community

Outcome and $\mathrm{T}$-time 
I -intervention refers to the parameters that were selected for investigation and /or the experimental parameters into the population studied $\mathrm{C}$ - are the factors that were used as controls, e.g., placebos
$\mathrm{O}$ - outcome refers to the outcome of the study and whether the results led to rejection or acceptance of the investigator's hypothesis

$\mathrm{T}$ - the time frame within which the study was conducted

\section{TABLE 2: ARTICLES INCLUDED IN THE STUDY}

$1^{\text {ST GROUP (POSITIVE ASSOCIATION- studies stating occlusion has a role in TMD ) }}$

\begin{tabular}{|c|c|c|c|c|c|c|}
\hline S.No & Author & Population (P) & Intervention (I) & $\begin{array}{l}\text { Comparison } \\
\text { (C) }\end{array}$ & Outcome (O) & $\begin{array}{l}\text { Time } \\
(\mathrm{T})\end{array}$ \\
\hline 1 & $\begin{array}{ll}\text { Haralur } & \text { SB } \\
\text { et.al., }^{18} & \end{array}$ & $\begin{array}{l}\mathrm{N}=100 \quad \mathrm{~N}=50 \\
\text { (asymptomatic), } \mathrm{N}= \\
50 \text { (symptomatic) }\end{array}$ & $\begin{array}{l}\text { Dynamic } \\
\text { occlusal contacts } \\
\text { evaluated by } \\
\text { conventional and } \\
\text { digital methods. } \\
\text { Centric, lateral } \\
\text { and protrusive } \\
\text { interferences by } \\
\text { a conventional } \\
\text { method } \\
\text { occlusion time } \\
\text { and disclusion } \\
\text { time by a digital } \\
\text { method }\end{array}$ & $\begin{array}{l}\mathrm{N}=50 \\
\text { asymptomatic } \\
\text { individuals }\end{array}$ & $\begin{array}{l}\text { There is } \\
\text { Association } \\
\text { between } \\
\text { dynamic } \\
\text { occlusal } \\
\text { parameters ( } \\
\text { centric slide, } \\
\text { balancing side } \\
\text { interference)and } \\
\text { TMD } \\
\text { There is an } \\
\text { association } \\
\text { between } \\
\text { prolonged } \\
\text { occlusion and } \\
\text { disclusion time } \\
\text { and TMD }\end{array}$ & \\
\hline 2 & $\begin{array}{l}\text { kirveskari p et } \\
\text { al., }{ }^{19}\end{array}$ & $\begin{array}{l}\text { Total } \mathrm{N}=62 \\
\text { Treatment group } \mathrm{N}= \\
33\end{array}$ & $\begin{array}{l}\text { Treatment group } \\
\text { occlusal } \\
\text { interferences like } \\
\text { the elimination } \\
\text { of slide in RCP- } \\
\text { MI and posterior }\end{array}$ & $\begin{array}{l}\text { Placebo group } \\
\mathrm{N}=32\end{array}$ & $\begin{array}{l}\text { Reduction in } \\
\text { subjective } \\
\text { symptoms } \\
\text { followed } \\
\text { modifications } \\
\text { of occlusion; }\end{array}$ & $2 \mathrm{yrs}$ \\
\hline
\end{tabular}




\begin{tabular}{|c|c|c|c|c|c|}
\hline & & & $\begin{array}{l}\text { contacts during } \\
\text { lateral and } \\
\text { protrusive } \\
\text { movements } \\
\text { Subjects in the } \\
\text { placebo group } \\
\text { had a mock } \\
\text { adjustment, } \\
\text { polishing of non- } \\
\text { occluding } \\
\text { fillings, i.e., n } \\
\text { occlusal changes }\end{array}$ & & $\begin{array}{l}\text { ergo, there is an } \\
\text { association } \\
\text { between } \\
\text { occlusal } \\
\text { interferences } \\
\text { and signs and } \\
\text { symptoms of } \\
\text { TMD }\end{array}$ \\
\hline 3 & Riolo ML et.al., ${ }^{20}$ & $\begin{array}{l}\mathrm{N}=1342 \\
\text { Age } 6-17 \\
\end{array}$ & $\begin{array}{l}\text { Analyzed for } \\
\text { specific types of } \\
\text { occlusion, } \\
\text { subjective } \\
\text { symptoms, and } \\
\text { clinical signs of } \\
\text { TMD }\end{array}$ & & $\begin{array}{l}\text { An association } \\
\text { exists between } \\
\text { specific features } \\
\text { of occlusion, } \\
\text { including open } \\
\text { bite, increased } \\
\text { overjet, sounds } \\
\text { in older } \\
\text { children with a } \\
\text { buccal crossbite }\end{array}$ \\
\hline 4 & $\begin{array}{l}\text { Forrsell } \mathrm{H} \text { et } \\
\text { al., }{ }^{21}\end{array}$ & $\begin{array}{l}\mathrm{N}=91 \text { patients with } \\
\text { headaches and signs } \\
\text { and symptoms of } \\
\text { TMD }\end{array}$ & $\begin{array}{l}\text { Occlusal } \\
\text { adjustments on } \\
48 \text { patients and } \\
\text { mock adjustment } \\
\text { on } 43 \\
19 \text { of the } 48 \\
\text { patients had } \\
\text { occlusal splints } \\
\text { as well }\end{array}$ & $\begin{array}{l}\mathrm{N}=43 \text { (control } \\
\text { group) patients } \\
\text { had a mock } \\
\text { adjustment }\end{array}$ & $\begin{array}{l}\text { Significantly } \\
\text { more reduction } \\
\text { in signs and } \\
\text { symptoms of } \\
\text { treatment group } \\
\text { independent od } \\
\text { splint therapy }\end{array}$ \\
\hline
\end{tabular}




\begin{tabular}{|c|c|c|c|c|c|}
\hline 5 & Kirveskariet.al., ${ }^{22}$ & $\begin{array}{l}\text { Treatment group } \\
\mathrm{N}=53\end{array}$ & $\begin{array}{l}\text { Elimination of } \\
\text { interferences } \\
\text { annually in the } \\
\text { treatment group } \\
\mathrm{N}=53\end{array}$ & $\begin{array}{l}\text { Control group } \\
\mathrm{N}=46 \\
\text { underwent } \\
\text { placebo } \\
\text { treatment }\end{array}$ & $\begin{array}{l}\text { A significant } \\
\text { association } \\
\text { between the } \\
\text { number of } \\
\text { interferences } \\
\text { and signs of } \\
\text { CM disorders }\end{array}$ \\
\hline 6 & Pullingeret.al., ${ }^{23}$ & $\mathrm{~N}=147$ asymptomatic & $\begin{array}{l}11 \text { occlusal } \\
\text { features anterior } \\
\text { open bite; } \\
\text { unilateral } \\
\text { maxillary lingual } \\
\text { crossbite; RCP- } \\
\text { ICP slide; } \\
\text { unilateral RCP } \\
\text { contact; } \\
\text { overbite; overjet; } \\
\text { dental midline } \\
\text { discrepancy; the } \\
\text { number } \\
\text { missing teeth; } \\
\text { the greater of the } \\
\text { mesiodistal } \\
\text { intermaxillary } \\
\text { relationship } \\
\text { discrepancies at } \\
\text { the first molar } \\
\text { intermaxillary } \\
\text { relationship(right } \\
\text { vs. } \\
\text { asymmetry). }\end{array}$ & $\begin{array}{l}\text { Disc } \\
\text { displacement } \\
\text { with reduction } \\
\text { (n=81), disc } \\
\text { displacement } \\
\text { without } \\
\text { reduction } \\
\text { (n=48), } \\
\text { osteoarthrosis } \\
\text { with disc } \\
\text { displacement } \\
\text { history } \\
\text { (n=75),primary } \\
\text { osteoarthrosis } \\
\text { (n=85), and } \\
\text { myalgia only } \\
\text { (n=124). }\end{array}$ & $\begin{array}{l}\text { Significant } \\
\text { associations } \\
\text { exist for each } \\
\text { disease. }\end{array}$ \\
\hline
\end{tabular}


$2^{\text {ND }}$ GROUP (NEGATIVE ASSOCIATION- studies stating there is no relation between occlusion and TMD )

\begin{tabular}{|c|c|c|c|c|c|c|}
\hline S.No & Author & $\begin{array}{l}\text { Population } \\
\text { (P) }\end{array}$ & Intervention (I) & $\begin{array}{l}\text { Comparison } \\
\text { (C) }\end{array}$ & Outcome $(\mathrm{O})$ & $\begin{array}{l}\text { Time } \\
(\mathrm{T})\end{array}$ \\
\hline 7 & $\begin{array}{l}\text { AlhajjMN et } \\
\text { al. }^{24}\end{array}$ & $\begin{array}{l}\mathrm{N}=\quad 625 \\
\text { (75\% female } \\
\text { ) age } 34.1 \\
+/-6.7 \\
\text { (divided into } \\
\text { 4groups) }\end{array}$ & 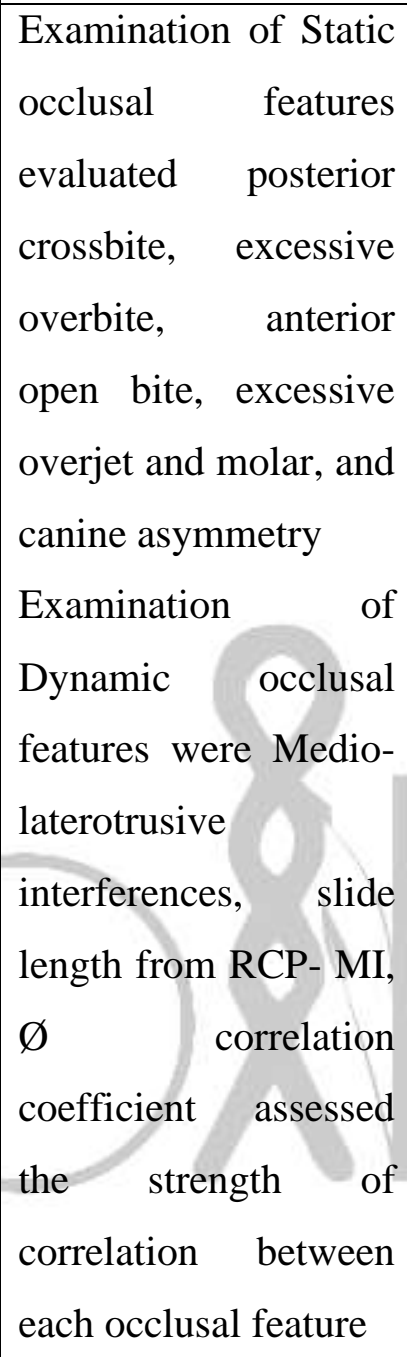 & $\begin{array}{l}\text { Data on } \\
\text { malocclusion } \\
\text { in the General } \\
\text { population }\end{array}$ & $\begin{array}{l}\text { In adult subjects with } \\
\text { or without signs and } \\
\text { symptoms of TMD, } \\
\text { static and dynamic } \\
\text { malocclusion were } \\
\text { comparably present } \\
\text { No association of } \\
\text { occlusion with TMD }\end{array}$ & \\
\hline 8 & $\begin{array}{l}\text { Droukas B et } \\
\text { al., }{ }^{25}\end{array}$ & $\begin{array}{l}\mathrm{N}=50 \\
37 \text { female } \\
13 \text { male }\end{array}$ & $\begin{array}{l}\text { Recording of occlusal } \\
\text { contacts in ICP, type } \\
\text { of contact - unilateral } \\
\text { or bilateral in RCP, } \\
\text { RCP- ICP distance in } \\
\text { the sagittal, vertical } \\
\text { and lateral direction, } \\
\text { types of occlusion - } \\
\text { contacts in }\end{array}$ & & $\begin{array}{l}\text { Even though occlusal } \\
\text { conditions varied } \\
\text { considerable and, no } \\
\text { association could be } \\
\text { established with signs } \\
\text { and symptoms of } \\
\text { mandibular } \\
\text { dysfunction }\end{array}$ & $\begin{array}{l}3 \\
\text { month } \\
\text { period }\end{array}$ \\
\hline
\end{tabular}




\begin{tabular}{|c|c|c|c|c|c|c|}
\hline & & & $\begin{array}{l}\text { laterotrusion and } \\
\text { protrusion and } \\
\text { occlusal interferences }\end{array}$ & & & \\
\hline 9 & $\begin{array}{l}\text { Takenoshita Y } \\
\text { et al., }\end{array}$ & $\begin{array}{l}\mathrm{N}=79 \\
\mathrm{~N}=44 \\
\text { control } \\
\text { group } \\
\mathrm{N}= \\
\text { treatment } \\
\text { group }\end{array}$ & $\begin{array}{l}\text { Wax bite was taken in } \\
\text { ICP and analyzed } \\
\text { electrophotometrically }\end{array}$ & $\begin{array}{l}\mathrm{N}=44 \text { control } \\
\text { group }\end{array}$ & $\begin{array}{l}\text { No association of } \\
\text { occlusal contact area } \\
\text { with signs and } \\
\text { symptoms of TMD }\end{array}$ & \\
\hline 10 & $\begin{array}{l}\text { De Boever JA } \\
\text { et al., }{ }^{27}\end{array}$ & $\begin{array}{lr}\mathrm{N}= & 135 \\
\text { patients }(33 \\
\text { male , } 102 \\
\text { female) } \\
\\
\end{array}$ & $\begin{array}{l}\text { Reassurance, occlusal } \\
\text { splint and } \\
\text { muscle exercise, } \\
\text { occlusal adjustment, } \\
\text { occlusal rehabilitation }\end{array}$ & $\begin{array}{l}\text { Intergroup } \\
\text { comparison }\end{array}$ & $\begin{array}{l}\text { No association } \\
\text { between the number } \\
\text { of occluding molars } \\
\text { and premolars and the } \\
\text { severity of the } \\
\text { symptoms and signs }\end{array}$ & $\begin{array}{l}12 \\
\text { months }\end{array}$ \\
\hline 11 & $\begin{array}{l}\mathrm{V} \text { a n d e r a s } \\
\mathrm{AP}^{28}\end{array}$ & $\begin{array}{l}\mathrm{N}=\quad 386 \\
\text { children age } \\
6-10 \text { yrs } \\
\text { With or } \\
\text { without } \\
\text { unpleasant } \\
\text { life events, } \\
\text { both females } \\
\mathrm{n} \text { males } \\
\mathrm{N}= \\
\text { calm group } \\
\mathrm{N}=105 \\
\text { subjects } \\
\text { with } \\
\text { unpleasant } \\
\text { life events }\end{array}$ & $\begin{array}{l}\text { Questionnaire to the } \\
\text { parents and followed } \\
\text { by clinical } \\
\text { examination }\end{array}$ & $\begin{array}{l}\mathrm{N}=250 \quad \text { calm } \\
\text { group } \\
\text { subjects } \\
\text { without } \\
\text { unpleasant life } \\
\text { events) }\end{array}$ & $\begin{array}{l}\text { No statistically } \\
\text { significant correlation } \\
\text { between each clinical } \\
\text { sign and each type of } \\
\text { morphological } \\
\text { malocclusion in both } \\
\text { groups } \\
\text { No } \\
\text { association/correlation } \\
\text { between malocclusion } \\
\text { and CMD }\end{array}$ & \\
\hline
\end{tabular}




\begin{tabular}{|c|c|c|c|c|}
\hline & & $\begin{array}{l}\text { (tense, } \\
\text { anxious) } \\
\text { (not calm ) } \\
\mathrm{N}=25 \\
\text { subjects } \\
\text { with } \\
\text { dentofacial } \\
\text { injuries } \\
\text { either with } \\
\text { or without } \\
\text { unpleasant } \\
\text { life events }\end{array}$ & & \\
\hline 12 & $\begin{array}{l}\text { Szentpetery et } \\
\text { al. }{ }^{29}\end{array}$ & $\begin{array}{l}\mathrm{N}=600 \\
\mathrm{~N}= \\
(\text { male) } \\
\mathrm{N}= \\
\text { (female) }\end{array}$ & $\begin{array}{l}\text { Assessed } \\
\text { morphological } \\
\text { occlusion viz., angle } \\
\text { classification of inter- } \\
\text { arch occlusal } \\
\text { relations, crowding, } \\
\text { deep bite, edge to } \\
\text { edge bite, open bite, } \\
\text { crossbite, tilting, and } \\
\text { over the eruption. } \\
\text { Functional occlusion, } \\
\text { balance, and hyper } \\
\text { balance }\end{array}$ & $\begin{array}{l}\text { No association of } \\
\text { TMD with } \\
\text { morphological or } \\
\text { functional occlusion }\end{array}$ \\
\hline 13 & Mohlin et al., ${ }^{30}$ & $\begin{array}{ll}\mathrm{N}= & 505 \\
\text { women } & \text { age } \\
\text { range }: & 20- \\
45 \mathrm{yrs} & \end{array}$ & $\begin{array}{l}\text { Clinical assessment of } \\
\text { TMJ movements, pain } \\
\text { on movement, TMJ } \\
\text { sounds, muscle } \\
\text { tenderness, TMJ } \\
\text { tenderness, and cuspal } \\
\text { interferences }\end{array}$ & $\begin{array}{l}\text { No association } \\
\text { between any variable } \\
\text { malocclusion } \\
\text { severity of } \\
\text { signs }\end{array}$ \\
\hline
\end{tabular}




\begin{tabular}{|c|c|c|c|c|c|}
\hline 14 & Jenni et al. ${ }^{31}$ & $\begin{array}{l}\mathrm{N}=210 \\
\text { (both female } \\
\text { and male ) } \\
\text { Age } \\
\text { between 20- } \\
69\end{array}$ & $\begin{array}{l}\text { Screening to evaluate } \\
\text { signs of TMD in } \\
\text { patients with and } \\
\text { without occlusal } \\
\text { interferences }\end{array}$ & $\begin{array}{l}\text { Individuals } \\
\text { without } \\
\text { occlusal } \\
\text { interferences }\end{array}$ & $\begin{array}{l}\text { No significant } \\
\text { association between } \\
\text { occlusal interferences } \\
\text { and TMD }\end{array}$ \\
\hline 15 & $\begin{array}{l}\text { Dworkin et al. } \\
32\end{array}$ & $\begin{array}{l}\mathrm{N}=\quad 108 \\
\text { symptomatic } \\
\text { patients }\end{array}$ & $\begin{array}{l}\text { Screening field } \\
\text { examination for signs } \\
\text { and symptoms of } \\
\text { TMD } \\
\text { Field characteristic- } \\
\text { pain report, } \\
\text { morphologic } \\
\text { occlusion }\end{array}$ & $\begin{array}{lr}\mathrm{N}= & 210 \\
\text { controls in two } \\
\text { age } & \text { group } \\
\text { strata } & 18-44 \\
\text { yrs, } & 45-75 y r s\end{array}$ & $\begin{array}{l}\text { No association } \\
\text { between any of the } \\
\text { variables evaluated } \\
\text { and signs and } \\
\text { symptoms of TMD }\end{array}$ \\
\hline 16 & $\begin{array}{l}\text { Seligman DA } \\
\text { et al., }\end{array}$ & $\begin{array}{l}124 \text { Female } \\
\text { patients with } \\
\text { intracapsular } \\
\text { TMD }\end{array}$ & $\begin{array}{l}\text { Nine occlusal factors } \\
\text { were used for } \\
\text { screening }\end{array}$ & $\begin{array}{l}\mathrm{N}=47 \\
\text { asymptomatic } \\
\text { female } \\
\text { controls with } \\
\text { an association } \\
\text { to } 9 \text { occlusal } \\
\text { factors } \\
\mathrm{N}=3 \text { attrition } \\
\text { severity } \\
\text { measures }\end{array}$ & $\begin{array}{lr}\text { Poor } & \text { association } \\
\text { between } & \text { TMD } \\
\text { patients and occlusal } \\
\text { and attritional factors }\end{array}$ \\
\hline 17 & $\begin{array}{l}\text { Pullinger et al., } \\
1988^{34}\end{array}$ & $\begin{array}{l}\mathrm{N}=220 \\
\text { Female } \\
\mathrm{N}=102 \\
\text { Male } \mathrm{N}= \\
120\end{array}$ & $\begin{array}{l}\text { They were assessed } \\
\text { for masticatory pain } \\
\text { and dysfunction by } \\
\text { questionnaire, clinical } \\
\text { examination, and } \\
\text { evaluation of dental } \\
\text { casts. } \\
\text { Assessment for } \\
\text { vertical overlap, }\end{array}$ & & $\begin{array}{l}\text { No association of } \\
\text { clicking with angle } \\
\text { class, deep bite, } \\
\text { length of RCP-ICP } \\
\text { slide, or unilateral } \\
\text { RCP contact. }\end{array}$ \\
\hline
\end{tabular}




\begin{tabular}{|c|c|c|c|c|c|}
\hline & & & $\begin{array}{l}\text { crossbite, ICP \& RCP } \\
\text { relationship unilateral } \\
\text { molar contact in RCP }\end{array}$ & & \\
\hline 18 & $\begin{array}{l}\text { McFarlane et } \\
\text { al., }{ }^{35}\end{array}$ & $\begin{array}{l}\mathrm{N}=196 \\
\text { healthy } \\
\text { subjects } \\
\text { aged 18-65 } \\
\text { years }\end{array}$ & $\begin{array}{l}\text { One occlusal feature } \\
\text { missing posterior } \\
\text { teeth }\end{array}$ & $\begin{array}{l}\mathrm{N}=131 \text { subjects } \\
\text { with pain } \\
\text { dysfunction } \\
\text { syndrome }\end{array}$ & $\begin{array}{l}\text { Multiple variables: no } \\
\text { association. }\end{array}$ \\
\hline 19 & DeSousaet.al., ${ }^{36}$ & $\begin{array}{l}\text { N=58 TMD } \\
\text { free subjects } \\
\text { aged > }>15 \\
\text { years }\end{array}$ & $\begin{array}{l}\text { Five occlusal features, } \\
\text { anterior open bite; } \\
\text { posterior crossbite; } \\
\text { overbite }>4 \mathrm{~mm} \text {; } \\
\text { overjet }>5 \mathrm{~mm} \text {; more } \\
\text { than } 5 \text { posterior teeth } \\
\text { were missing. }\end{array}$ & $\begin{array}{ll}\mathrm{N}=42 & \mathrm{TMD} \\
\text { subjects } & \text { aged } \\
>15 & \end{array}$ & $\begin{array}{l}\text { Single variable: no } \\
\text { association }\end{array}$ \\
\hline 20 & $\begin{array}{l}\text { Manfredini et } \\
\text { al., }{ }^{37}\end{array}$ & $\begin{array}{l}\mathrm{N}=58 \mathrm{TMD} \\
\text { free subjects } \\
\text { (aged 20-40 } \\
\text { years) } \\
\text { without a } \\
\text { history of } \\
\text { orthodontics }\end{array}$ & $\begin{array}{l}\text { Three occlusal } \\
\text { features canine class, } \\
\text { molar } \\
\text { asymmetry. }\end{array}$ & $\begin{array}{l}\text { N=96 TMD } \\
\text { patients (aged } \\
20-40 \text { years) } \\
\text { without a } \\
\text { history of } \\
\text { orthodontics }\end{array}$ & $\begin{array}{l}\text { Single variable: no } \\
\text { association. }\end{array}$ \\
\hline
\end{tabular}

$3^{\text {RD }}$ GROUP ( studies stating the requirement of further studies to assess the role of occlusion in TMD)

\begin{tabular}{|c|c|c|c|c|c|c|}
\hline S.No & Author & Population (P) & Intervention (I) & $\begin{array}{l}\text { Comparison } \\
\text { (C) }\end{array}$ & Outcome $(\mathrm{O})$ & $\begin{array}{l}\text { Time } \\
(\mathrm{T})\end{array}$ \\
\hline 21 & $\begin{array}{l}\text { Le Bell Y et } \\
\text { al., }{ }^{38}\end{array}$ & $\begin{array}{l}\mathrm{N}=26 \text { healthy } \\
\text { women } \\
\mathrm{N}=21 \text { women } \\
\text { with a previous } \\
\text { history of TMD }\end{array}$ & $\begin{array}{l}\text { Divided into treatment } \\
\text { and placebo group } \\
\text { Artificial } \\
\text { interferences were } \\
\text { created in the } \\
\text { treatment groups and }\end{array}$ & $\begin{array}{l}\text { Compared } \\
\text { with placebo } \\
\text { groups of } \\
\text { each group }\end{array}$ & $\begin{array}{l}\text { Patients with a } \\
\text { previous history } \\
\text { of TMD were } \\
\text { more associated } \\
\text { with signs and } \\
\text { symptoms of }\end{array}$ & $\begin{array}{l}2-16 \\
\text { months }\end{array}$ \\
\hline
\end{tabular}




\begin{tabular}{|c|c|c|c|c|c|}
\hline & & & $\begin{array}{l}\text { simulated in the } \\
\text { placebo group, i.e., } \\
\text { each of the } 2 \text { groups } \\
\text { was subdivided into a } \\
\text { treatment group and } \\
\text { placebo group } \\
\text { Patients without a } \\
\text { history of } \mathrm{TMD} \text { : } \\
\mathrm{N}=14 \text { placebo, } \mathrm{N}=12 \\
\text { treatment group } \\
\text { Patients with history } \\
\text { of } \mathrm{TMD}: \mathrm{N}=11 \\
\text { placebo, } \mathrm{N}=10 \\
\text { treatment group }\end{array}$ & & $\begin{array}{l}\text { TMD in the } \\
\text { presence of } \\
\text { occlusal } \\
\text { interference } \\
\text { Further studies } \\
\text { required to } \\
\text { know the } \\
\text { association }\end{array}$ \\
\hline 22 & $\begin{array}{l}\text { Abd Al Hadi L } \\
39\end{array}$ & $\begin{array}{l}600 \text { men and } \\
\text { women } \\
\text { Age: } 16-22 \text { yrs } \\
\mathrm{N}=\quad 300 \\
\text { symptomatic }\end{array}$ & \begin{tabular}{lr} 
Assessed 5 & occlusal \\
parameters & angles \\
class, type & of \\
eccentric & occlusion, \\
chewing & side \\
preference, & and \\
working and non- \\
\multicolumn{2}{l}{ working interferences }
\end{tabular} & $\begin{array}{l}\mathrm{N}=\quad 300 \\
\text { asymptomatic } \\
\text { patients }\end{array}$ & $\begin{array}{l}\text { Various levels } \\
\text { of association } \\
\text { and non- } \\
\text { association }\end{array}$ \\
\hline
\end{tabular}

RESULTS:

The search (both physical and electronic) resulted in 41 citations, of which 19 were excluded based on the exclusion criteria mentioned elsewhere.

Thus 22articles were selected, which were in accordance with the objectives of the research question

A full-text retrieval was accomplished by $1^{\text {st }}$ author of the included papers, and an agreement as regards their inclusion/exclusion was arrived at by discussion among the $1^{\text {st }}, 2^{\text {nd }}$ authors. The study findings were categorized by $3^{\text {rd }}$ and $4^{\text {th }}$ author as follows:

STUDY FINDINGS:

The study findings could be categorized as under randomized control trials $\mathrm{N}=2$, Casecontrol studies $N=6$, prevalence studies $\mathrm{N}=9$.

The selected articles were discussed with the fifth author. He suggested that since the topic chosen for the review has high variable opinions and conclusions, 
systematic quantitative analysis is not possible, and it has to be assessed qualitatively, where meta-analysis is not possible to attain mean and standard deviation values.

In summary, the pattern of associations could be divided into 3 groups.

Gl $(N=6)$ studies which did find an association with occlusal features and TMD

G2, the major group ( $N=14)$, consisted of studies that failed to find any association between occlusal factors and TMD.

$\mathrm{G} 3(N=2)$ questioned some of the findings of $\mathrm{Gl}$, stating a lack of methodological rigor in them.

\section{DISCUSSION:}

Temporomandibular disorders have a contentious history with regard to history. Among the many etiologic factors suggested, the one that was and still remains still unresolved despite decades of research has been the association between occlusal factors and temporomandibular joint disorders, and since the occlusion is of primary importance to the dental profession, it is essential that the role of occlusion be clearly defined in its relation to temporomandibular disorders-failing which the profession will have negligible if any role in the management of temporomandibular disorders. Ever since James B Costen first described a group of conditions that later was eponymously called Costen's syndrome, the dental fraternity has been intensely searching for an etiology for the condition. ${ }^{1,2}$

Despite the existence of huge literature about TMD and occlusion, there is still controversy and contradictory opinions on the interaction of TMD and occlusion. Currently, the term temporomandibular disorders are the most frequently used term to represent disturbances and dysfunction of the stomatognathic system. Through a series of workshops and symposia, the term temporomandibular disorders became the "Gold standard" for the disorders' diagnostic criteria. In four different entities, the term occlusion is used in the dental literature: 1) the anatomic jaw relation (orthodontic): Angle classification, 2) static contact between the teeth of the upper and lower jaws, 3) dynamic contact between the teeth of the upper and lower jaws, and 4) the prosthetic classifications, more specifically, the complete/ incomplete dentition versus complete dentitions and the presence of fixed/ removable prosthesis. ${ }^{44}$

Table 2 summarizes in the PICOT format the results of the reviewing 22articles. The table reveals that while there is little to substantiate the role of occlusion in TMD, it is only a partial reflection of reality. This is in contradistinction to the conclusions of many authors because there are studies that do find the association of occlusal features with TMD and also articles which question the methodological rigor of studies in G2.

The conclusions drawn from the literature are: 1) the role of occlusion in the etiology "TMD" is not absolutely assessed. 2) occlusal interferences affect TMD, 3) "TMD" is multifactorial; subsequently, it will be affected by different treatment modalities ( the biopsychological model modalities of illness approach), 4) adaptation is an important quality of the 2021 April - May Special Edition | www.jbino.com | Innovative Association 
human being; it is more specific to the stomatognathic system. ${ }^{44}$ This manuscript reviewed the literature on the association between features of dental occlusion and temporomandibular disorders. Based on findings, which support the absence of disease-specific association, there is no ground to hypothesize a significant role for dental association in the pathophysiology of TMDs. Dental clinicians are thus encouraged to move forward and abandon the old-fashioned gnathological paradigm. The authors are therefore of the opinion that since the majority of studies do not demonstrate a role for occlusion in $T M D$, it would be prudent for the clinician managing TMDs to adopt a conservative reversible approach, e.g., occlusal appliances, bio-feedback, etc. rather than adopt aggressive irreversible interventional strategies like orthodontics or extensive occlusal rehabilitation; till such time as

\section{REFERENCES:}

1.

Okeson P. Management disorders of temporomandibular occlusion. $7^{\text {th }}$ ed; Elsevier publications Mosby: 2013.

2. Dawson PE. Functional occlusion from TMJ to smile design. Elsevier publications Mosby: 2007.

3. Egermark I, Thilander B. Craniomandibular disorders with special reference to orthodontic treatment: An evaluation from childhood to adulthood. Am J OrthodDentofacOrthop 1992;101:28-34.

4. Fuchs P. The muscular activity of the chewing apparatus during night sleep. J Oral Rehab 1975; 2: 35-8.

5. Wadhwa L, Ashok Utreja A, Tewar A. A study of clinical signs and symptoms of further studies definitely confirms the role of occlusion in TMD.

The main limitations of the study are the articles were assessed qualitatively, but quantitative analysis of the literature would have been helped in obtaining a perfect outcome with clinical significance.

CONCLUSION:

Due to the phenomenon of the multiple catch-all or container concepts of both temporomandibular disorders and occlusion, there are different options to research. This systematic review, therefore, arrives at the following conclusions:

1. A majority of studies do not find occlusion as an aetiologic role in TMD

2.there are a few studies that, however, find an association

This may enable the clinician and the researcher a springboard to do further study on the topic.

temporomandibular dysfunction in subjects with normal occlusion, untreated, and treated malocclusions. Am J OrthodDentofacOrthop 1993;103:54-61.

6. Trenouth MJ. The relationship between bruxism and temporomandibular joint dysfunction as shown by computer analysis of nocturnal tooth contact patterns. J Oral Rehab 1979; 6: 81-7.

7. Pullinger AG, Seligman DA. The Degree to Which Attrition Characterizes Differentiated Patient Groups of Temporomandibular Disorder. J Orofacial Pain 1993;7:196-208.

8. Stringert HG, Worms FW. Variations in skeletal and dental patterns in patients with structural and functional alterations of the temporomandibular joint: A preliminary report. Am J Orthod 89: 285-297, 1986. 
9. Riise C, Sheikholesla A. Influence of experimental interfering occlusal contacts on the activity of the anterior temporal and masseter muscles during mastication. .J Oral Rehab 1984; 11: 325-333.

10. Pullinger AG, Seligman DA. Overbite and overjet characteristics of refined diagnostic groups of temporomandibular disorder patients. Am J OrthodDentofacOrthop 1991;100: 401-15.

11.Rugh JD, Barghi N, Drago CJ. Experimental occlusal discrepancies and nocturnal bruxism. J Prosthet Dent 1984; 51 (4): 548-53.

12. Westling L. Occlusal interferences in retruded contact position and temporomandibular joint sounds. J Oral Rehab 1995 22; 601-606.

13. Tsolka P, Walter JD, Wilson RF, Preiskel HW. Occlusal variables, bruxism, and temporomandibular disorders: a clinical and kinesiographic assessment J Oral Rehab 1995;22: 849-856.

14. Suvinen TI, Kemppaine P. Review of clinical EMG studies related to muscle and occlusal factors in healthy and TMD subjects. J Oral Rehab 2007;34: 631-644.

15. Luther F. TMD and occlusion part I. Damned if we do? Occlusion: the interface of dentistry and orthodontics. Br Dent $J$ 2007; 202: 1-7.

16. Tanne K, Tanaka E, Sakuda M. Association Between Malocclusion and Temporomandibular Disorders in Orthodontic Patients Before Treatment. J Orofacial Pain 1993;7:156-162.

17. Marbach JJ, Raphael KG,Dohrenwend BP, Lennon MC. The validity of tooth grinding measures: etiology of pain dysfunction syndrome revisited. JADA, 1990; 120: 32733.
18. Haralur SB. Digital Evaluation of Functional Occlusion Parameters and their Association with Temporomandibular Disorders. J Clin Diag Res. 2013; 7(8): 1772775.

19. Kirveskari $P$, Le Bell $Y$, Salonen M, Forssell $H$, Grans L. Effect of elimination of occlusal interferences on signs and symptoms of the craniomandibular disorder in young adults. J Oral Rehab 1989;16: 21-6.

20. Riolo ML, Brandt D,TenHave TH. Associations between occlusal characteristics and signs and symptoms of TMJ dysfunction in children and young adults. Am

OrthodDentofacOrthop 1987;92:487-77.

21. Forssell $H$, Kirveskari $P$, Kangasniemi $P$. Effect of occlusal adjustment on mandibular dysfunction. A double-blind study. Acta OdontolScand1986;44:63-69.

22. Kirveskari $P$, Alanen $P, T$. Jamai. Association between craniomandibular disorders and occlusal interferences.JProsthet Dent 1989;61:62:66-9.

23. Pullinger AG, Seligman DA, Gornbein JA. A multiple logistic regression analysis of the risk and relative odds of temporomandibular disorders as a function of common occlusal features. J Dent Res. 1993;72:968-979.

24. Alhajj M N, Khalifa N, Abduo J, Amran A G, Ismai I A. Determination of occlusal vertical dimension for complete dentures patients: an updated review. J Oral Rehab 2017; 44: 896-907.

25. Droukas B, Lindee C, Carlsson GE. Occlusion and mandibular dysfunction: $A$ clinical study of patients referred for functional disturbances of the masticatory system. J Prosthet Dent 1985; 53(3): 402-06. 
26. Takenoshita Y, Ikebe T, Yamamoto M, Oka M. Occlusal contact area and temporomandibular joint symptoms. OralSurc Oral Med Oral Pathol 1991;72:388-94.

27. DeBoever JA, Adriaens PA. Occlusal relationship in patients with paindysfunction symptoms in the temporomandibular joints. J Oral Rehab 1983; 10: 1-7.

28. Vanderas AP. Relationship between craniomandibular dysfunction and malocclusion in white children with and without unpleasant life events. J Oral Rehab 1994; 21:177-183.

29. Szentpetery A, Fazekas A, Mari A: An epidemiologic study of mandibular dysfunction dependence on different variables. Community Dent Oral Epidemiol 1987; 15: 164-68.

30. Mohlin E, Prevalence of mandibular dysfunction and relation between malocclusion and mandibular dysfunction in a group of women in Sweden. Eur $J$ Orthod 1983:5:1 15-123.

31. Jenni M, Schurch E, Geering AH. Symptoms of functional disorders in the masticatory system-An epidemiological study [in German], SchweiiMonatsschrZahnmed 1987:97: 1357-365.

32. Dworkin SF, Huggins KH, LCResche L, et al. Epidemiology of signs and symptoms in temporomandibular disorders: Clinical signs in cases and controls. J Am Dent Assoc 1990:120:273-81.

33. Seligman DA, Pullinger AG. Analysis of occlusal variables, dental attrition, and age for distinguishing healthy controls from female patients with intracapsular temporomandibular disorders. J Prosthet Dent 2000;83:76-82.
34. Pullinger AG, Seligman DA, Solberg WK. Temporomandibular disorders. Part II: Occlusal factors associated with temporomandibular joint tenderness and dysfunction. J Prosthet Dent 1988; 59(3): 363-67.

35. Macfarlane TV, Gray RJM, Kincey J, Worthington HV. Factors associated with temporomandibular disorder, pain dysfunction syndrome (PDS): a manchester case-control study. Oral Dis. 2001;7:321-30.

36. de Sousa ST, de Mello VV, Magalh aes BG, de Assis Morais MP, Vasconcelos MM, de Franc a Caldas Junior A et al. The role of occlusal factors on the occurrence of temporomandibular disorders. Cranio2015;33:211-16.

37. Manfredini D, Lombardo L, Siciliani G. Dental angle class asymmetry and temporomandibular OrofacOrthop. 2017:78:253-58.

38. Le Bell Y, JamsaT, Korri S, Niemi PM, Alanen P. Effect of artificial occlusal interferences depends on previous experience of temporomandibular disorders. Acta OdontolScand2002;60:219-22.

39.L.Abd Al-Hadi L. Prevalence of temporomandibular disorders in relation to some occlusal parameters. J Prosthet Dent 1993;70:345-50.

40.Kampe T, Carlsson GE, Hannerz $H$, Haraldson T. Three-year longitudinal study of mandibular dysfunction in young adults with intact and restored dentitions. Acta OdontolScand 1987;45:25-30.

41. Forssell H, Kirveskari P, Kangasniemi P. Effect of occlusal adjustment on mandibular dysfunction. A double-blind study. Acta OdontolScand 1986;44:63-9.

42. MCNamara JA, Seligman DA, Okeson JP. Occlusion, Orthodontic Treatment, and 
Temporomandibular Disorders: A Review. J Orofacial Pain 1995:9:73-90.

43. Sheikholeslam A, Riise JC. Influence of experimental interfering occlusal contacts on the activity of the anterior temporal and masseter muscles during submaximal and maximal bite in the intercuspal position. J Oral Rehab 1983;10:207-14.

44. de Kanter RJAM, Battistuzzi PGFCM, Truin G. Temporomandiblardisorders : "occlusion" matters!. Pain Res Manage 2018;1-13. 